\title{
Thalidomide inhibits proliferation and epithelial-mesenchymal transition by modulating CD133 expression in pancreatic cancer cells
}

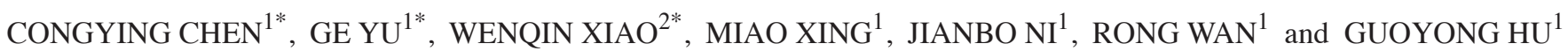 \\ ${ }^{1}$ Department of Gastroenterology, Shanghai General Hospital, \\ Shanghai Jiaotong University School of Medicine, Shanghai 200080; ${ }^{2}$ Department of Gastroenterology, \\ Shanghai Tenth People's Hospital, Tongji University School of Medicine, Shanghai 200072, P.R. China
}

Received June 5, 2016; Accepted July 27, 2017

DOI: $10.3892 /$ ol.2017.7213

\begin{abstract}
Pancreatic cancer is a solid malignancy with a high mortality rate, on account of the high incidence of metastasis at the time of detection. The aggressiveness of pancreatic cancer may be partly driven by cancer stem cells (CSCs), which are characterized by the ability to self-renew and recapitulate tumors in the ectopic setting. However, although a number of drugs targeting CSCs are currently under clinical investigation, few effective drugs have been developed. The present study demonstrated that thalidomide inhibited cell proliferation and metastasis in pancreatic cancer cell lines through the inhibition of epithelial mesenchymal transition. The effect of thalidomide was more pronounced in cluster of differentiation 133 (CD133) ${ }^{+}$SW1990 cells than in Capan-2 cells, in which CD133 expression was almost undetectable. The results revealed that CD133 is likely to serve a role in the antitumor effect of thalidomide and indicated that thalidomide could be developed as a CSC-specific adjuvant chemotherapy in pancreatic cancer.
\end{abstract}

\section{Introduction}

Pancreatic cancer is a disease with a high mortality rate that is characterized by the early metastasis to local and distant organs; the majority of patients present with unresectable disease at the time of initial diagnosis (1). Therefore, patients

Correspondence to: Dr Guoyong Hu, Department of Gastroenterology, Shanghai General Hospital, Shanghai Jiaotong University School of Medicine, 100 Haining Road, Shanghai 200080, P.R. China

E-mail: guoyonghu@aliyun.com

*Contributed equally

Key words: thalidomide, pancreatic cancer, proliferation, metastasis, epithelial-mesenchymal transition, cancer stem cells, cluster of differentiation 133 with pancreatic cancer have a poor prognosis, with an overall 5 -year survival rate of $<5 \%$ in the United States between 1975 and 2008 (2). No significant advances in the treatment of pancreatic cancer have been made in $>10$ years, largely due to of the resistance of the disease to conventional chemotherapy and radiation therapy (3-6). The involvement of cancer stem cells (CSC; also termed tumor-initiating cells) in the development of chemotherapy resistance has been reported in a number of types of malignancy (7-9). CSCs are a phenotypically distinct population of cells that are functionally defined by their ability to form tumors, self-renew and differentiate, as well as their resistance to chemotherapy $(10,11)$. Therefore, the development of novel chemotherapeutic agents that are effective against CSCs is urgently required.

Thalidomide is a non-barbiturate sedative and hypnotic drug with anti-angiogenic and immunomodulatory properties (12). Thalidomide is currently used in the treatment of various types of malignant tumor, including prostate cancer (13), glioblastoma (14), glioma (15), renal cell carcinoma (16) and advanced breast cancer (17). The mechanism of action of thalidomide includes the inhibition of vascular endothelial growth factor, basic fibroblast growth factor and tumor necrosis factor- $\alpha$, the inhibition of angiogenesis, and the stimulation of natural killer cells (12). However, to the best of our knowledge, the use of thalidomide in the treatment of pancreatic cancer has not been assessed and the potential mechanism of thalidomide-mediated inhibition of tumor cell viability remains to be elucidated. In the present study, the effect of thalidomide on pancreatic cancer cells was examined, alongside its mechanism of action.

\section{Materials and methods}

Cell lines and reagents. The human pancreatic cancer SW1990, Capan-2, Panc-1, Patu8988 and Aspc-1 cell lines were purchased from the American Type Culture Collection (Manassas, VA, USA) and maintained in high-glucose Dulbecco's modified Eagle medium (DMEM) supplemented with $10 \%$ fetal bovine serum (FBS) (Gibco; Thermo Fisher Scientific, Inc., Waltham, MA, USA) and $1 \%$ penicillin-streptomycin (Gibco; Thermo Fisher Scientific, Inc.) at $37^{\circ} \mathrm{C}$ in a 
humidified incubator with $5 \% \mathrm{CO}_{2}$. Thalidomide was obtained from Selleck Chemicals (Houston, TX, USA). The MTT reagent and anti-cluster of differentiation 133 (CD133) antibody for western blotting were purchased from Sigma-Aldrich (Merck KGaA, Darmstadt, Germany; cat. no. SAB2107606). The phycoerythrin-conjugated CD133 antibody for flow cytometry was purchased from MiltenyiBiotec, Inc., (Auburn, CA, USA; cat. no. 130-080-801). Transwell filter inserts were obtained from Corning (Corning In corporated, Corning, NY, USA). Rabbit anti-E-cadherin (cat. no. sc-7870), rabbit anti-N-cadherin (cat. no. sc-7939), and mouse anti- $\beta$-actin (cat. no. sc-47778) were purchased from Santa Cruz Biotechnology, Inc., (Dallas, TX, USA). Thalidomide was dissolved in dimethyl sulfoxide (DMSO) to a storage concentration of $50 \mathrm{mmol} / \mathrm{l}$. An equal amount $(0.1 \% \mathrm{v} / \mathrm{v})$ of DMSO was present in all the treatment groups, including the control.

Cell proliferation assay. Cell viability was measured using the MTT assay, according to the manufacturer's protocol. Cells were seeded at a density of $5 \times 10^{4}$ cells/well in 96 -well flat-bottom microtiter plates. Cells were treated with different concentrations $(0,6.25,12.5,25,50$ or $100 \mu \mathrm{mol} / \mathrm{l})$ of thalidomide in $200 \mu \mathrm{l}$ complete medium for 24,48 or $72 \mathrm{~h}$ at $37^{\circ} \mathrm{C}$ in an incubator. Cells were then incubated with MTT $(0.25 \mathrm{mg} / \mathrm{ml})$ for $2-4 \mathrm{~h}$ at $37^{\circ} \mathrm{C}$. The formazan crystals in the cells were solubilized with a solution containing $50 \%$ dimethylformamide and $20 \%$ SDS (pH 4.7). Finally, the level of MTT/formazan was determined by measuring absorbance at a wavelength of $570 \mathrm{~nm}$ using a microplate reader (SPECTRA; Tecan Group, Ltd., Mannedorf, Switzerland).

Cell migration assays. SW1990 and Capan-2 cells were plated in 6 -well plates with various concentrations of thalidomide $(0$, $50,100 \mu \mathrm{mol} / \mathrm{l})$. At $48 \mathrm{~h}$, the cells were harvested, washed once in PBS and resuspended in serum-free high-glucose DMEM. A $200-\mu 1$ aliquot of cell suspension was added to Transwell filter inserts, resulting in a density of $2 \times 10^{4}$ cells per insert. High-glucose DMEM containing $10 \%$ FBS was added to the lower wells. Migration was allowed to proceed for $48 \mathrm{~h}$ at $37^{\circ} \mathrm{C}$. Cells that did not migrate through the filters were removed using cotton swabs, and cells that migrated through the inserts were fixed and stained with $1 \%$ crystal violetat room temperature. The number of migrating cells was observed with phase contrast microscopy (magnification, x200) and images were captured.

Flow cytometry. SW1990 and Capan-2 cells were plated in 6 -well plates for $48 \mathrm{~h}$ with various concentrations of thalidomide $(0,50,100 \mu \mathrm{mol} / \mathrm{l})$, then were harvested, washed twice in cold PBS and incubated with a phycoerythrin-conjugated CD133 antibody $(1: 10)$ for $30 \mathrm{~min}$ at $4^{\circ} \mathrm{C}$. Mouse IgG1-phycoerythrin (1:10; cat. no. IC002P; R\&D Systems, Inc.) was used as an isotype control antibody. Non-viable cells were eliminated with 7-aminoactinomycin D (Nanjing KeyGen Biotech Co., Ltd., Nanjing, China; cat. no. KGA219) staining. The labeled cells were analyzed by a BD FASCCanto II flow cytometer 338960 (BD Biosciences, Franklin Lakes, NJ, USA) in accordance with the manufacturer's protocol and data was analyzed using FlowJo v10 software (Tree Star, Inc., Ashland, OR, USA). Gating was implemented on the basis of negative-control staining profiles.
Table I. Polymerase chain reaction primer sequences.

Gene Primer sequence, 5'-3'

$\begin{array}{ll}\text { CD133 } & \\ \text { Forward } & \text { TTCTTGACCGACTGAGACCCA } \\ \text { Reverse } & \text { TCATGTTCTCCAACGCCTCTT } \\ \text { E-cadherin } & \\ \text { Forward } & \text { TCGACACCCGATTCAAAGTGG } \\ \text { Reverse } & \text { GTGGGTTATGAAACCGTAGAGG } \\ \text { N-cadherin } & \\ \text { Forward } & \text { AGCCAACCTTAACTGAGGAGT } \\ \text { Reverse } & \text { GGCAAGTTGATTGGAGGGATG } \\ \beta \text {-actin } & \\ \text { Forward } & \text { CTGGAACGGTGAAGGTGACA } \\ \text { Reverse } & \text { AAGGGACTTCCTGTAACAATGCA }\end{array}$

CD133, cluster of differentiation 133.
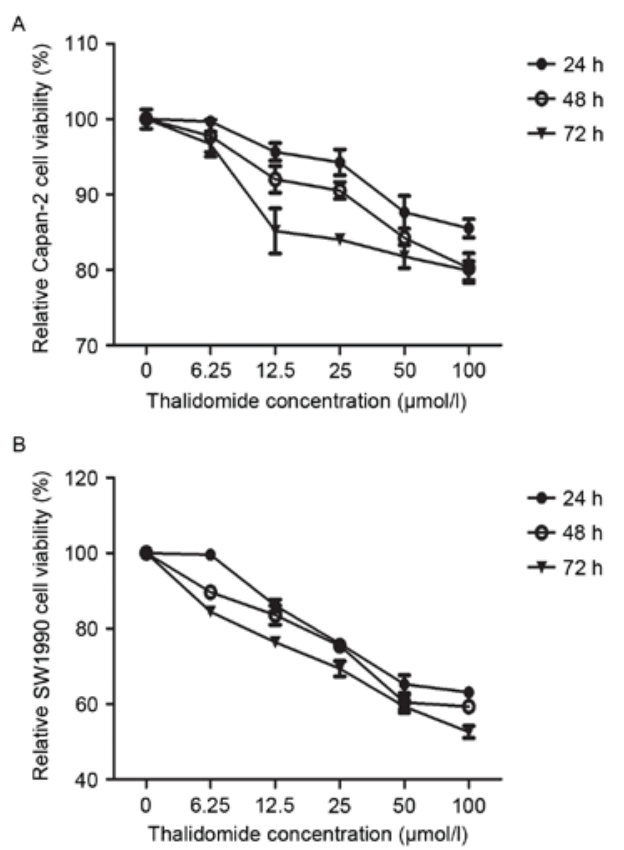

Figure 1. Thalidomide inhibits the cell viability of pancreatic cancer cell lines. The viability of (A) Capan-2 and (B) SW1990 cells treated with a range of thalidomide concentrations was measured with an MTT assay and compared with untreated cells. Thalidomide treatment induced a dose- and time-dependent inhibitionof cell viability. Results represent the mean \pm standard deviation from six independent experiments.

Reverse transcription-quantitative polymerase chain reaction (RT-qPCR) analysis. A density of 10×104 SW1990 and Capan-2 cells were respectively treated with $100 \mu \mathrm{mol} / 1$ thalidomide for $24 \mathrm{~h}$ and harvested, and total RNA was extracted using TRIzol (Invitrogen; Thermo Fisher Scientific, Inc.) according to the manufacturer's protocol, and subjected to reverse transcription using a PrimeScript RT reagent kit (Takara Bio, Inc., Otsu, Japan). The mRNA expression was detected by qPCR with an ABI PRISM ${ }^{\circledR} 7900$ HT Sequence Detection system (Applied Biosystems; Thermo Fisher Scientific, Inc.) using SYBR-Green Dye (Takara Bio, Inc.). 

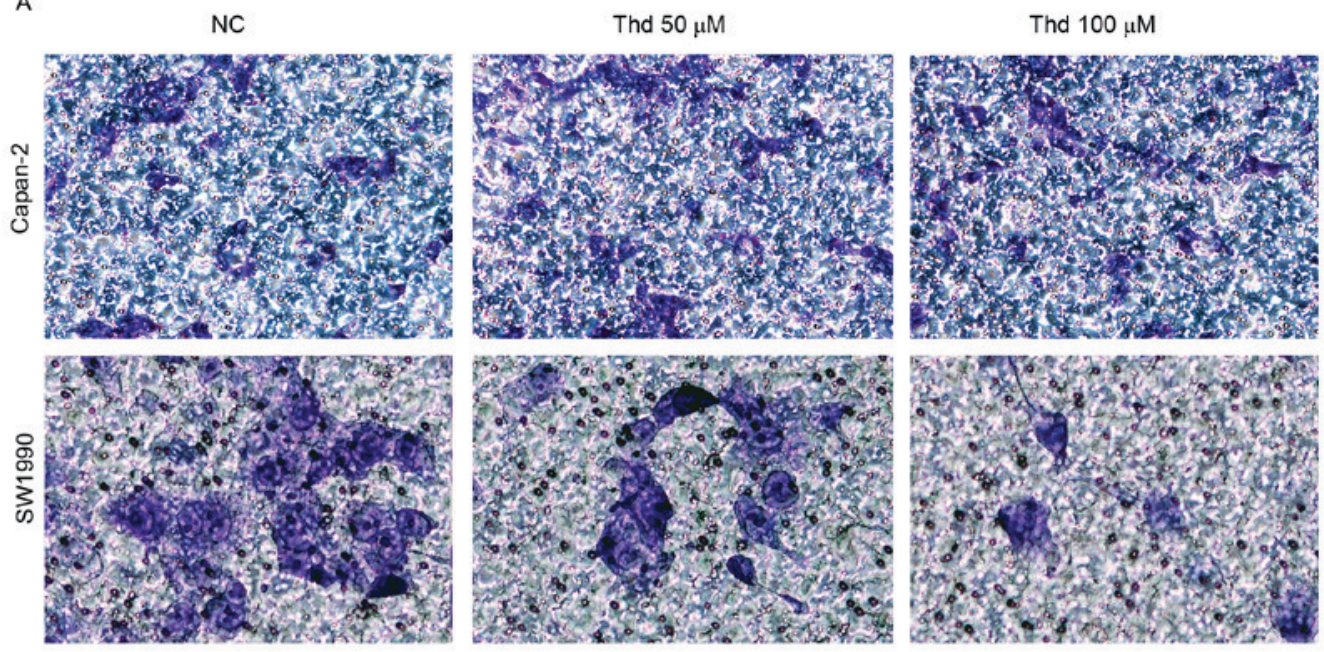

B

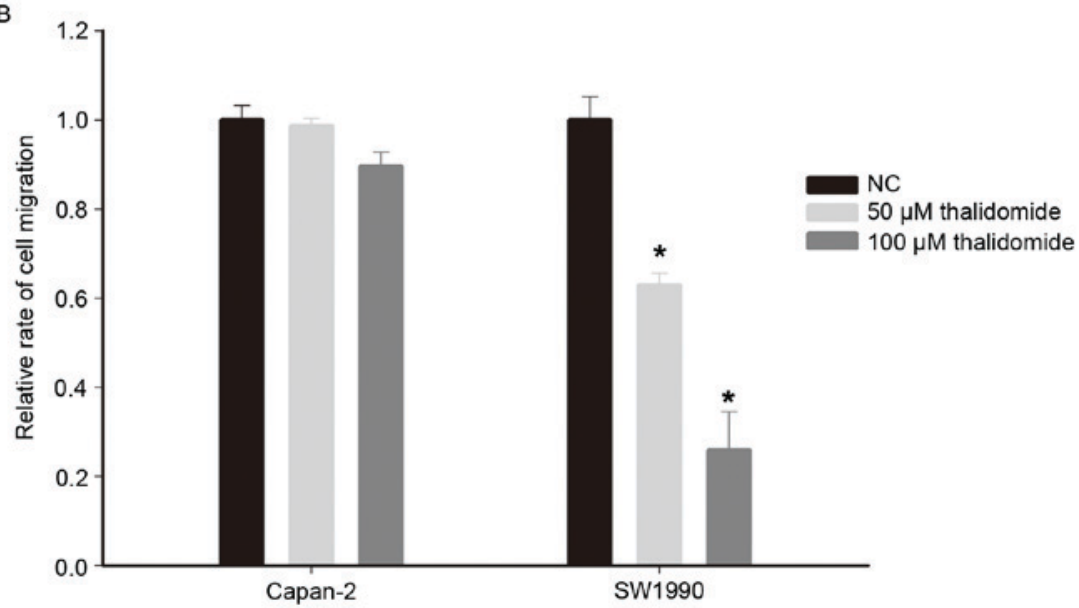

Figure 2. The effect of thalidomide on the migration of pancreatic cancer cell lines was assessed using Transwell filter inserts. (A) The relative proportion of migrated pancreatic cancer cells treated with thalidomide for $48 \mathrm{~h}$ was determined. Magnification, $\mathrm{x} 200$. (B) Analysis of cell migration. * $\mathrm{P}<0.05 \mathrm{compared}$ with NC. NC, negative control.

Primers used for qPCR are listed in Table I. cDNA was amplified with 40 PCR cycles $\left(0.5 \mathrm{sec}\right.$ at $95^{\circ} \mathrm{C} ; 10 \mathrm{sec}$ at $60^{\circ} \mathrm{C} ; 10 \mathrm{sec}$ at $72^{\circ} \mathrm{C}$ ). The relative expression levels were calculated using the $2^{-\Delta \Delta \mathrm{Cq}}$ method (18). All experiments were repeated at $\geq 3$ times.

Western blotting. SW1990 and Capan-2 cells were treated with $100 \mu \mathrm{mol} / 1$ thalidomide for $24 \mathrm{~h}$ and rinsed twice in PBS. The cells were harvestedand centrifuged at 2,000 $\mathrm{x}$ g for $5 \mathrm{~min}$ at $4^{\circ} \mathrm{C}$, then lysed for $2 \mathrm{~h}$ in radioimmunoprecipitation analysis lysis buffer (Beyotime Institute of Biotechnology, Haimen, China; cat. no. P0013B) on ice, and centrifuged at $12,000 \mathrm{x} \mathrm{g}$ for $10 \mathrm{~min}$ at $4^{\circ} \mathrm{C}$. The protein concentration was determined by using a bicinchoninic acid protein assay $\left(\mathrm{BCA}^{\mathrm{TM}}\right.$ protein assay kit; Pierce; Thermo Fisher Scientific, Inc.). Aliquots of $40 \mu \mathrm{g}$ protein were separated by $6 \%$ SDS-PAGE and transferred onto polyvinylidene fluoride membranes. Non-specific binding was blocked with $5 \%$ low-fat milk at room temperature for $1 \mathrm{~h}$ in a covered container. The membranes were incubated with the relevant primary antibodies overnight at $4{ }^{\circ} \mathrm{C}$ (dilutions: E-cadherin, 1:200; N-cadherin, 1:400, CD133, 1:800; $\beta$-actin, 1:1,000). The membranes were washed in PBS with $0.1 \%$ Tween-20 and incubated with the appropriate HRP-conjugated secondary antibodies (goat anti-rabbit IgG-HRP; 1:2,000; cat. no. sc-2004; goat anti-mouse IgG-HRP; 1:2,000; cat. no. sc-2005; Santa Cruz Biotechnology, Inc.) for $1 \mathrm{~h}$ at $37^{\circ} \mathrm{C}$. The blots were developed using an enhanced chemiluminescence (ECL)-detection system (Santa Cruz Biotechnology, Inc., Dallas, TX, USA), dried, and exposed to ECL film. All experiments were repeated $\geq 3$ times, with similar results.

Statistical analysis. SPSS 18.0 software (SPSS, Inc., Chicago, IL, USA) was used for statistical analysis. Data were obtained from three independent experiments and expressed as the mean \pm standard deviation. The statistical analyses performed included $\chi^{2}$ test and one-way analysis of variance (ANOVA), which was followed by Student-Newman-Keuls (SNK) as a post hoc test. $\mathrm{P}<0.05$ was considered to indicate a statistically significant difference.

\section{Results}

Thalidomide inhibits the proliferation of pancreatic cancer cells. The inhibitory effect of thalidomide on the growth of human pancreatic cancer cell lines was determined by an 
A
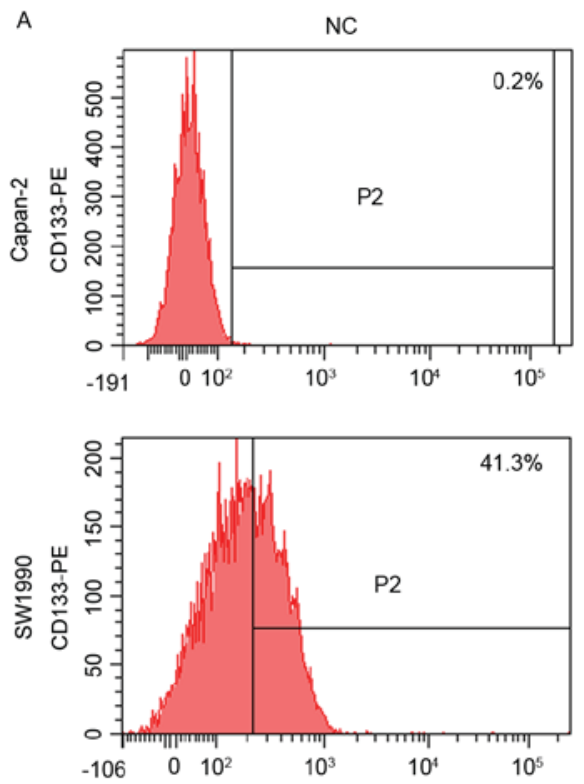
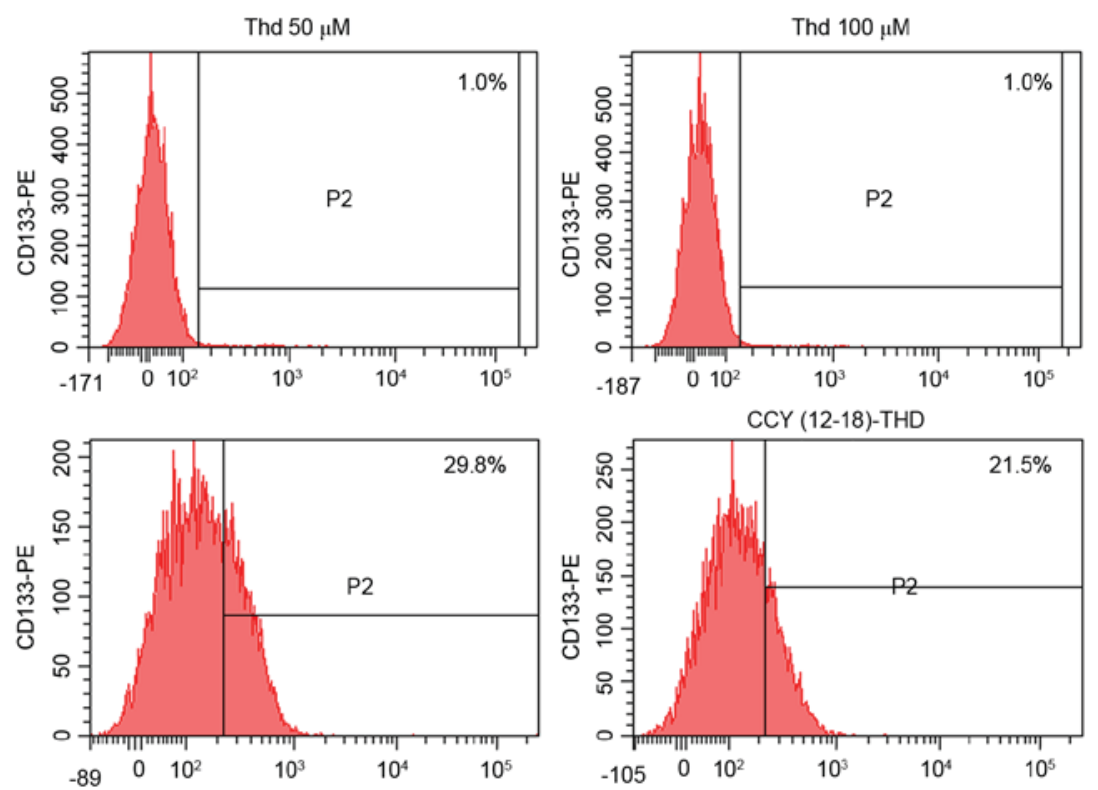

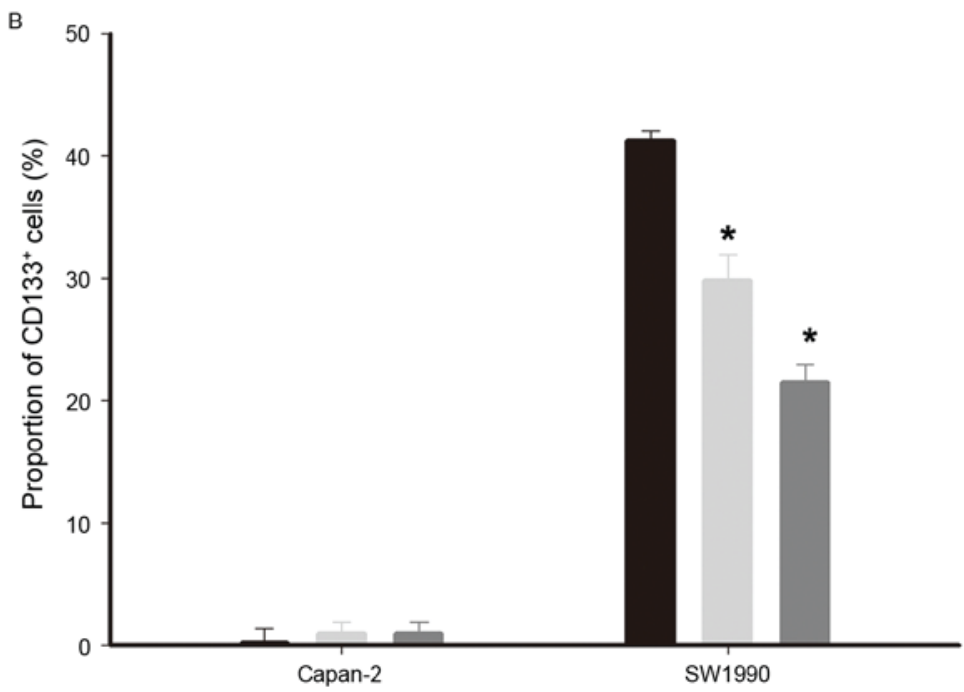

Figure 3. Flow cytometry analysis of the proportion of CD133 ${ }^{+}$cells in the Capan- 2 and SW1990 cell lines following treatment with different concentrations of thalidomide. (A) Capan- 2 cells were $0.2 \%$ CD133 ${ }^{+}$, compared with $41.3 \%$ for SW1990 cells. (B) Thalidomide treatment reduced the CD133 ${ }^{+}$cell subpopulation in SW1990 cells; however, there was no effect on Capan-2 cells. " $\mathrm{P}<0.05$ compared with NC. CD133, cluster of differentiation 133; NC, negative control.

MTT assay. In Capan-2 cells treated with thalidomide, a dose-dependent decrease in cell number was observed, with a 4-14, 7-19 and 14.8-20\% inhibition in cell growth, respectively (Fig. 1A). Thalidomide caused acomparatively higher doseand time-dependent inhibition of cell growth in SW1990 cells, with $13-36,16-40$, and $23-47 \%$ of growth at 24,48 and $72 \mathrm{~h}$ at 6.25-100 $\mu \mathrm{mol} / 1$, respectively (Fig. 1B). These data indicated that the mechanism for growth inhibition by thalidomide may differ between the two cell lines.

The proliferation of three other pancreatic cancer cell lines (Panc-1, Patu8988 and Aspc-1) was assessed in preliminary experiments using the MTT assay (data not shown). Results obtained from this analysis demonstrated that the proliferation of these three pancreatic cancer cell lines did not differ significantly following administration of thalidomide, whereas the proliferation of the Capan-2 and SW1990 cell lines were inhibited following treatment. Therefore, Capan-2 and SW1990 cells were selected for the present study.
Effects of thalidomide on migration in pancreatic cancer cells. To examine the effect of thalidomide on the motility of pancreatic cancer cells, a cell migration assay was conducted with Transwell filter inserts. The motility of SW1990 cells treated with thalidomide for $48 \mathrm{~h}$ was reduced compared withthe control cells (Fig. 2A and B; $\mathrm{P}<0.05$ ). However, the Capan-2 cells exposed to thalidomide treatment exhibited no significant decrease in motility (Fig. 2A and B), which was consistent with the pattern of growth inhibition in the two cell lines.

Thalidomide reduces the proportion of $C D 133^{+}$cell subpopulations in vitro. CD133 is a cell surface marker of CSCs in pancreatic cancer $(19,20)$. The effect of thalidomide on the proportion of pancreatic cancer cells with the CD133+ antigenic phenotype was analyzed by flow cytometry. The proportion of $\mathrm{CD} 133^{+}$cells in the Capan-2 and SW1990 lines was different, with $<1 \%$ of cells exhibiting CD133-positivity 
in the Capan-2 cell line compared with $>40 \%$ in SW1990. This indicated that the SW1990 line has a higher proportion of CSCs than the Capan-2 line. The proportion of $\mathrm{CD}_{133^{+}}$cells in the Capan-2 cell line were not significantly altered in response to thalidomide treatment (negative control, $0.2 \%$ vs. thalidomide, $1.0 \%$ ), whereas thalidomide treatment significantly decreased the proportion of $\mathrm{CD}_{133^{+}}$cells in the SW1990 cell line (negative control, $41.3 \%$ vs. thalidomide $50 \mu \mathrm{mol} / 1,29.8 \%$ and $100 \mu \mathrm{mol} / 1,21.5 \%$; Fig. 3; P<0.05).

Thalidomide inhibits EMT in pancreatic cancer cells. The dysregulation of the EMT program contributes to tumor initiation, invasion and metastatic spread, and is associated with an increase in tumor cell stemness (21). The teratogenic effect of thalidomide is well-documented; EMT governs morphogenesis and is activated during embryogenesis (22). Therefore, the EMT status was assessed in cells exposed to thalidomide. The primary molecular feature of cancer cells undergoing EMT is the upregulation of characteristic mesenchymal genes, including N-cadherin and E-cadherin (23). On the basis of the previous results that thalidomide could reduce the proportion of $\mathrm{CD}_{133^{+}}$cells, the expression of $\mathrm{N}$-cadherin, E-cadherin and CD133 were detected by RT-qPCR and western blotting.

The results of western blotting indicated that the protein expression of CD133 in Capan-2 cells was markedly lower than in SW1990 cells. Thalidomide treatment downregulated CD133 and N-cadherin, and upregulated E-cadherin mRNA and protein expression in SW1990 cells (Fig. 4). However, thalidomide did not significantly affect the expression of E-cadherin, N-cadherin and CD133 mRNA in Capan-2 cells. Therefore, it was concluded that thalidomide inhibited the EMT program in $\mathrm{CD}_{133^{+}}$cells, whereas it exhibited no significant effect in cells with low CD133 expression.

\section{Discussion}

Pancreatic cancer is the fourth-leading cause of cancer-associated mortality in the United States (24). Unlike other types of cancer, the survival rate of patients with this disease has not improved substantially in $\sim 40$ years, largely due to its aggressiveness (25). Emerging evidence indicates that the aggressiveness of pancreatic cancer may be partly driven by CSCs, phenotypically distinct cell populations $(10,26,27)$. CD133 is the most commonly expressed CSC marker in several cancer types, including pancreatic cancer (18,19,28-30).

In the present study, it was demonstrated that thalidomide inhibits the growth of human pancreatic cancer cells in vitro. However, the sensitivity to thalidomide differed between SW1990 and Capan-2 cells, which exhibit different levels of CD133 expression. The SW1990 cell line, in which the proportion of $\mathrm{CD}_{133^{+}}$cells was $>40 \%$, was more sensitive to thalidomide than the Capan- 2 cell line, of which $<1 \%$ were $\mathrm{CD} 133^{+}$cells, as demonstrated by the increased inhibition of cell growth and migration. These results indicated that the sensitivity of pancreatic cancer cells to thalidomide may dependent on the expression of CD133, and therefore, on the proportion of CSCs. Thalidomide reduced the $\mathrm{CD}_{133^{+}}$cell subpopulation in the SW1990 cell line and downregulated the mRNA and protein expression of CD133. Taken together, the results indicated that thalidomide may inhibit the proliferation and metastasis of
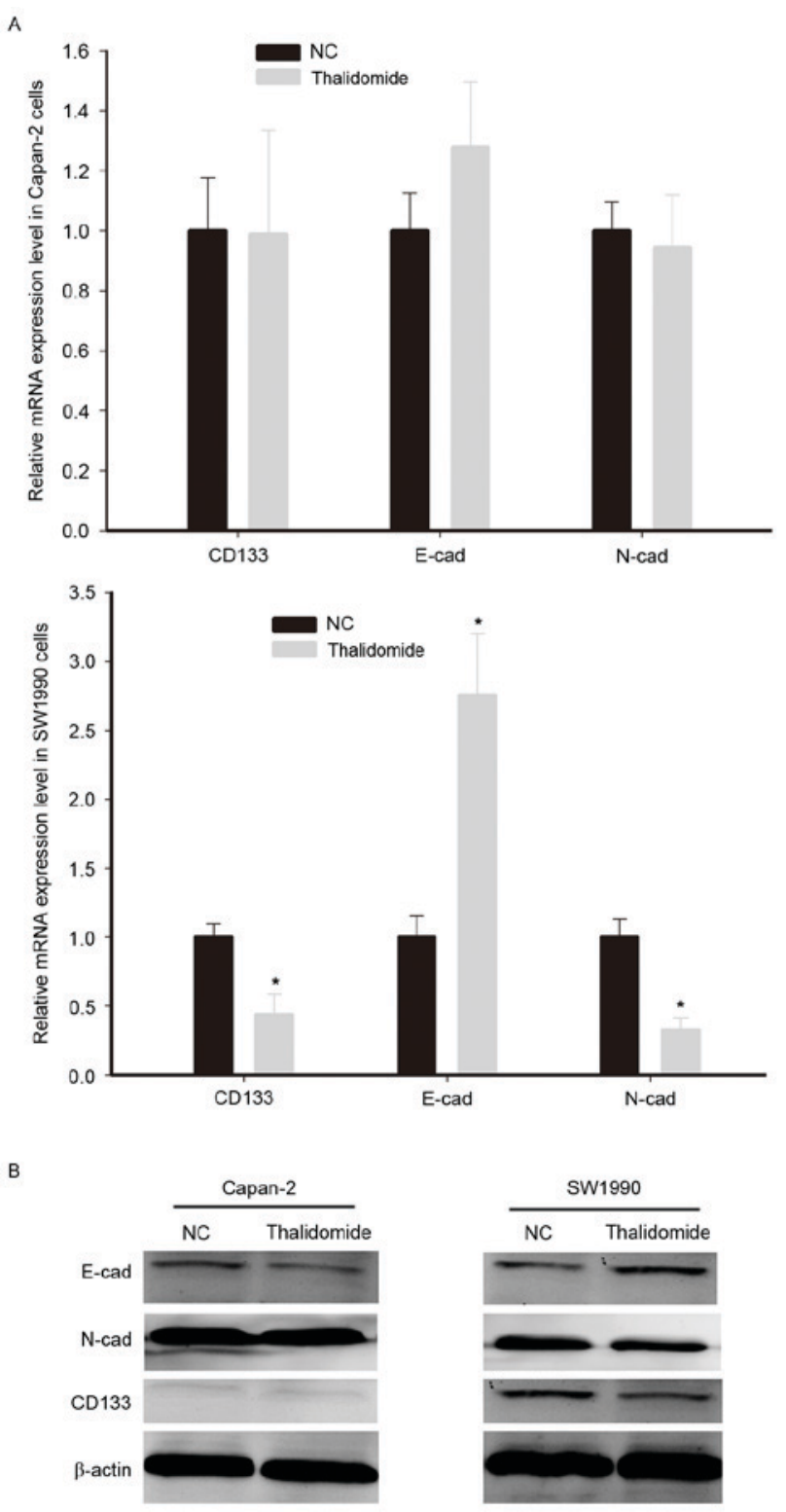

Figure 4. Thalidomide inhibited EMT and downregulated CD133 expression in SW1990 cells. The expression levels of E-cad, N-cad and CD133 were detected by (A) reverse transcription-quantitative polymerase chain reaction and (B) western blotting. Thalidomide downregulated CD133 and $\mathrm{N}$-cad mRNA and protein expression and upregulated E-cad mRNA and protein expression in SW1990 cells; however, it had no effect on the levels of these markers in Capan- 2 cells. " $\mathrm{P}<0.05$ compared with NC. EMT, epithelial-mesenchymal transition; CD133, cluster of differentiation 133; E-cad, E-cadherin; N-cad, N-cadherin; NC, negative control.

pancreatic cancer cells partly by modulating the expression of CD133, which suggests that the antitumor effects of thalidomide are partially mediated by inhibiting CSCs.

The epithelial-mesenchymal transition (EMT) is a process implicated in drug resistance, metastasis and the generation of CSCs $(21,31,32)$. The present study analyzed the mRNA and protein expression of the EMT markers E-cadherin and $\mathrm{N}$-cadherin in pancreatic cancer cells treated with thalidomide. The results of this analysis revealed that thalidomide upregulated E-cadherin expression and downregulated $\mathrm{N}$-cadherin expression in SW1990 cells, whereas there was no significant effect on these markers in Capan- 2 cells. These results 
suggested that thalidomide inhibited EMT in SW1990 cells and not in Capan-2 cells. Previous studies have demonstrated that CD133 is part of the regulatory network that facilitates EMT; CD133 is a transmembrane protein associated with the signaling pathways regulating the EMT program, particularly the cadherin switch $(28,33)$. In liver cancer cells, CD133+ cells exhibited upregulated $\mathrm{N}$-cadherin expression and downregulated E-cadherin expression compared with $\mathrm{CD}^{-3} 3^{-}$cells, which indicates that EMT occurs more frequently in CD133+ cells than in CD133- cells (34). Ding et al (29) reported that CD133 serves an important role in the regulation of $\mathrm{N}$-cadherin and Slug expression. However, other molecules are involved in the regulation of EMT, in addition to CD133. Further study is required to characterize this regulatory loop, which implies that thalidomide may inhibit EMT at least partly through the modulation of CD133.

In summary, the data of the present study indicate that the in vitro efficacy of thalidomide at inhibiting pancreatic cancer cell growth and migration is mediated by the downregulation of CD133 and the inhibition of EMT. The efficacy of thalidomide was dependent on the expression of CD133, as demonstrated by the different sensitivity to thalidomide of the SW1990 and Capan-2 pancreatic cancer cell lines, which express different levels of CD133. The results indicated that thalidomide may partly target CSCs. However, the differential response of SW1990 and Capan-2 cells to thalidomide warrants further investigation at the molecular level in order to further characterize the mechanisms underlying their sensitivity to thalidomide. Furthermore, assessing the effect of a combination of CSC-targeting and non-CSC-targeting therapies could be of value in optimizing the tumor response, enhancing long-term disease control and ultimately improving patient survival rates.

\section{Acknowledgements}

The present study was supported in part by grants from the National Natural Science Foundation of China (grant nos. 81372643 and 81270543) and Foundation for Shanghai Science and Technology Committee (grant no. XBR2013082). The funders had no role in study design, data collection and analysis, the decision to publish, or preparation of the study.

\section{References}

1. Vincent A, Herman J, Schulick R, Hruban RH and Goggins M: Pancreatic cancer. Lancet 378: 607-620, 2011.

2. Siegel R, Naishadham D and Jemal A: Cancer statistics, 2013. CA Cancer J Clin 63: 11-30, 2013.

3. Conroy T, Desseigne F, Ychou M, Bouché O, Guimbaud R, Bécouarn Y, Adenis A, Raoul JL, Gourgou-Bourgade S, de la Fouchardière $\mathrm{C}$, et al: FOLFIRINOX versus gemcitabine for metastatic pancreatic cancer. N Engl J Med 364: 1817-1825, 2011.

4. Von Hoff DD, Ramanathan RK, Borad MJ, Laheru DA, Smith LS, Wood TE, Korn RL, Desai N, Trieu V, Iglesias JL, et al: Gemcitabine plus nab-paclitaxel is an active regimen in patients with advanced pancreatic cancer: A phase I/II trial. J Clin Oncol 29: 4548-4554, 2011.

5. Forssell H, Pröh K, Wester M and Krona H: Tumor size as measured at initial X-ray examination, not length of bile duct stricture, predicts survival in patients with unresectable pancreatic cancer. BMC Cancer 12: 429, 2012.
6. Hirono S, Kawai M, Okada KI, Miyazawa M, Shimizu A, Kitahata Y, Ueno $M$ and Yamaue H: Treatment strategy for borderline resectable pancreatic cancer with radiographic artery involvement. Pancreas 45: 1438-1446, 2016.

7. Sharma VP, Anderson NT and Geusz ME: Circadian properties of cancer stem cells in glioma cell cultures and tumorspheres. Cancer Lett 345: 65-74, 2014

8. Hale JS, Sinyuk M, Rich JN and Lathia JD: Decoding the cancer stem cell hypothesis in glioblastoma. CNS Oncol 2: 319-330, 2013.

9. Huang Y, Ju B, Tian J, Liu F, Yu H, Xiao H, Liu X, Liu W, Yao Z and Hao Q: Ovarian cancer stem cell-specific gene expression profiling and targeted drug prescreening. Oncol Rep 31: 1235-1248, 2014.

10. Gilbertson RJ and Graham TA: Cancer: Resolving the stem-cell debate. Nature 488: 462-463, 2012.

11. Rosen JM and Jordan CT: The increasing complexity of the cancer stem cell paradigm. Science 324: 1670-1673, 2009.

12. Stewart AK: Medicine. How thalidomide works against cancer. Science 343: 256-257, 2014

13. Song L, Zhou X and Li X: Phase II trial of granulocyte-macrophage colony-stimulating factor plus thalidomide in older patients with castration-resistant prostate cancer. Mol Clin Oncol 3: 865-868, 2015

14. Milanovic D, Sticht C, Röhrich M, Maier P, Grosu AL and Herskind C: Inhibition of 13-cis retinoic acid-induced gene expression of reactive-resistance genes by thalidomide in glioblastoma tumours in vivo. Oncotarget 6: 28938-28948, 2015.

15. Ruiz J, Case D, Enevold G, Rosdhal R, Tatter SB, Ellis TL, McQuellon RP, McMullen KP, Stieber VW, Shaw EG and Lesser GJ: A phase II trial of thalidomide and procarbazine in adult patients with recurrent or progressive malignant gliomas. J Neurooncol 106: 611-617, 2012.

16. Tunio MA, Hashmi A, Qayyum A, Naimatullah N and Masood R: Low-dose thalidomide in patients with metastatic renal cell carcinoma. J Pak Med Assoc 62: 876-879, 2012.

17. Burris HA III, Jones SF, Shipley D, Meluch AA, Greco FA, Barton JH, Yardley DA and Hainsworth JD: Phase II study of capecitabine in combination with thalidomide in patients with metastatic breast cancer. Cancer Invest 28: 408-412, 2010.

18. Livak KJ and Schmittgen TD: Analysis of relative gene expression data using real-time quantitative PCR and the 2(-Delta Delta C(T)) method. Methods 25: 402-408, 2001.

19. Hermann PC, Huber SL, Herrler T, Aicher A, Ellwart JW, Guba M, Bruns CJ and Heeschen C: Distinct populations of cancer stem cells determine tumor growth and metastatic activity in human pancreatic cancer. Cell Stem Cell 1: 313-323, 2007.

20. Lee HJ, You DD, Choi DW, Choi YS, Kim SJ, Won YS and Moon HJ: Significance of CD133 as a cancer stem cell markers focusing on the tumorigenicity of pancreatic cancer cell lines. J Korean Surg Soc 81: 263-270, 2011.

21. Thiery JP: Epithelial-mesenchymal transitions in tumour progression. Nat Rev Cancer 2: 442-454, 2002.

22. Lyons JG, Lobo E, Martorana AM and Myerscough MR: Clonal diversity in carcinomas: Its implications for tumour progression and the contribution made to it by epithelial-mesenchymal transitions. Clin Exp Metastasis 25: 665-677, 2008.

23. Mani SA, Guo W, Liao MJ, Eaton EN, Ayyanan A, Zhou AY, Brooks M, Reinhard F, Zhang CC, Shipitsin M, et al: The epithelial-mesenchymal transition generates cells with properties of stem cells. Cell 133: 704-715, 2008

24. Wiseman DA, Werner SR and Crowell PL: Cell cycle arrest by the isoprenoids perillyl alcohol, geraniol, and farnesol is mediated by p21(Cip1) and p27(Kip1) in human pancreatic adenocarcinoma cells. J Pharmacol Exp Ther 320: 1163-1170, 2007.

25. Guillaumond F, Iovanna JL and Vasseur S: Pancreatic tumor cell metabolism: Focus on glycolysis and its connected metabolic pathways. Arch Biochem Biophys 545: 69-73, 2014.

26. Antoniou A, Hébrant A, Dom G, Dumont JE and Maenhaut C: Cancer stem cells, a fuzzy evolving concept: A cell population or a cell property? Cell Cycle 12: 3743-3748, 2013.

27. Kumar R, Dholakia A and Rasheed Z: Stem cell-directed therapies in pancreatic cancer. Curr Probl Cancer 37: 280-286, 2013.

28. Ding Q, Yoshimitsu M, Kuwahata T, Maeda K, Hayashi T, Obara T, Miyazaki Y, Matsubara S, Natsugoe S and Takao S: Establishment of a highly migratory subclone reveals that CD133 contributes to migration and invasion through epithelial-mesenchymal transition in pancreatic cancer. Hum Cell 25: 1-8, 2012. 
29. Ding Q, Miyazaki Y, Tsukasa K, Matsubara S, Yoshimitsu M and Takao S: CD133 facilitates epithelial-mesenchymal transition through interaction with the ERK pathway in pancreatic cancer metastasis. Mol Cancer 13: 15, 2014.

30. Weng CC, Kuo KK, Su HT, Hsiao PJ, Chen YW, Wu DC, Hung WC and Cheng KH: Pancreatic tumor progression associated with CD133 overexpression: Involvement of increased TERT expression and epidermal growth factor receptor-dependent Akt activation. Pancreas 45: 443-457, 2016.

31. Fan YL, Zheng M, Tang YL and Liang XH: A new perspective of vasculogenic mimicry: EMT and cancer stem cells (Review) Oncol Lett 6: 1174-1180, 2013.
32. Hao J, Zhang Y, Deng M, Ye R, Zhao S, Wang Y, Li J and Zhao Z: MicroRNA control of epithelial-mesenchymal transition in cancer stem cells. Int J Cancer 135: 1019-1027, 2014.

33. Cai C, Yu JW, Wu JG, Lu RQ, Ni XC, Wang SL and Jiang BJ: CD133 promotes the invasion and metastasis of gastric cancer via epithelial-mesenchymal transition. Zhonghua Wei Chang Wai Ke Za Zhi 16: 662-667, 2013 (In Chinese).

34. Na DC, Lee JE, Yoo JE, Oh BK, Choi GH and Park YN: Invasion and EMT-associated genes are up-regulated in B viral hepatocellular carcinoma with high expression of CD133-human and cell culture study. Exp Mol Pathol 90: 66-73, 2011. 